九州大学学術情報リポジトリ

Kyushu University Institutional Repository

\title{
Mechanistic Insights into Nanobubble Merging Studied Using In Situ Liquid-Phase Electron Microscopy
}

Sarthak, Nag

Department of Mechanical Engineering, Kyushu University

Tomo, Yoko

Department of Mechanical Engineering, Kyushu University

Takahashi, Ko j i

Department of Aeronautics and Astronautics, Kyushu University

Kohno, Masamichi

Department of Mechanical Engineering, Kyushu University

http://hdl. handle. net/2324/4151256

出版情報: Langmuir，2021-01-05. American Chemical Society バージョン：

権利関係 : 


\title{
Mechanistic insight into nanobubble merging
}

\section{studied using in-situ liquid phase electron}

\section{microscopy}

\author{
Sarthak Nag, ${ }^{\dagger, *}$ Yoko Tomo, ${ }^{\dagger}$ Koji Takahashi, ${ }^{\text {t,* }}$ Masamichi Kohno ${ }^{\dagger, *, *}$ \\ ${ }^{\dagger}$ Department of Mechanical Engineering, Kyushu University, Fukuoka 819-0395, Japan \\ ${ }^{\ddagger}$ Department of Aeronautics and Astronautics, Kyushu University, Fukuoka 819-0395, Japan \\ *International Institute for Carbon-Neutral Energy Research (WPI-I2CNER), Kyushu University, \\ Fukuoka 819-0395, Japan \\ *Corresponding Author: kohno@mech.kyushu-u.ac.jp
}

KEYWORDS: nanobubbles; merging; gas layer; liquid phase electron microscopy.

\begin{abstract}
Nanobubbles have attracted great interest in recent times due to their application in water treatment, surface cleaning and targeted drug delivery, yet the challenge remains to gain thorough understanding of their unique behavior and dynamics for their utilization in numerous potential
\end{abstract}


applications. In this work, we have used liquid phase electron microscopy technique to gain insights into the quasistatic merging of surface nanobubbles. The electron beam environment was controlled in order to suppress any new nucleation and slow down the merging process. The transmission electron microscopy (TEM) study reveals that merging of closely positioned surface nanobubbles is initiated by gradual localized changes in the physical properties of the region between adjoining nanobubble boundary. The observed phenomenon is then analyzed and discussed based on the different perceptions: localized liquid density gradient and bridge formation for gas exchange. In this study, it is estimated that the merging of the stable nanobubbles is initiated by the formation of thin gas layer. This work not only enhances our understanding of the merging process of stable surface nanobubbles but will also lead to exploring new domains for nanobubble application.

\section{INTRODUCTION}

Understanding of the fluid transport and the interfacial behavior at nanoscale has been of prime importance for the advancements in emerging technologies like rapid heat dissipation, water desalination, self-cleaning surfaces and targeted drug delivery. ${ }^{1-3}$ Nanobubbles, the ultrafine gas domains present in the bulk liquid and at the solid-liquid interfaces, ${ }^{4-6}$ have gained interest in the last decade due to their numerous existing and prospective applications in chemical, biological and interfacial science domains. ${ }^{7-11}$ Much of the focus towards the nanobubbles has been due to their distinctive properties like long-span metastability (metastable because of their questioned stability based on excessive Laplace pressure $)^{4,7}$ and very high surface to volume ratio, ${ }^{5,12}$ which makes them desirable for application in drug delivery, ${ }^{13}$ decontamination of surfaces and liquids, ${ }^{14}$ biomedical imaging, ${ }^{15,16}$ nanomaterial fabrication ${ }^{7,17}$ and soil fertility and crop production 
enhancement. ${ }^{18}$ Nanobubbles also play a key role in various thermal-fluid sciences related phenomena like nucleation of boiling, ${ }^{19}$ microdroplets nucleation, ${ }^{20}$ enhancement of wall-slip, ${ }^{21}$ etc.

The existence of nanobubbles and their longevity has been long debated due to "coexisting" high Laplace pressure and high dissolution times, ${ }^{22-25}$ and even classical thermodynamics have failed to explain this longevity and stability. ${ }^{4,26}$ At the same time, this also questions the applicability of macroscale continuum theories at the nanoscopic length scales ${ }^{22}$ and increasing dominance of interactions at nanoscale. The long-term stability of nanobubbles has been hypothesized with numerous stability theories: contamination theory, ${ }^{27}$ dynamic equilibrium theory, ${ }^{28}$ contact line pinning $^{4,26}$ are some of them. However, none of these theories have been accepted without problems to explain the nanobubbles' peculiar stability. ${ }^{7}$ The observation of nanobubbles and their dynamics is intrinsically difficult due to their ultra-small size and the limitations in existing experimental instrumentation and techniques. Recently, the utilization of TEMs to image liquids has been made possible due to advances in nanofabrication technology and facile liquid-cell fabrication methods. ${ }^{29,30}$ This has enabled the opportunity for observation of fluids and their interfaces directly with tens of nanometer resolution and millisecond time resolution, ${ }^{31}$ which would not have been possible with previously adopted atomic force microscopy or optical microscopy. ${ }^{26,32,33}$ Recent advancements in the imaging techniques like super resolution confocal microscopy has allowed to observe nanobubbles in an explicit non-invasive environment, ${ }^{34}$ but the resolution remains low due to the diffraction limit of light.

Liquid phase electron microscopy (LPEM) involves encapsulation of the fluid of interest between two electron transparent sheets that allows imaging inside the vacuum column of TEM without evaporation. ${ }^{35}$ Additionally, observing water (or protic liquids) in liquid cell allows the 
observation of real time in-situ nucleation of nanobubbles due to radiolysis as well as succeeding nanoscale phenomena and interactions. ${ }^{36}$ So far, the focus of the nanobubbles' research using LPEM has been on evaluating the critical size of the stable nanobubbles, ${ }^{31,37}$ the growth regimes, ${ }^{38}$ collapse,$^{39}$ and interactions between the nanobubbles. ${ }^{40}$ The interaction between the neighboring nanobubbles and study of the interfaces is one aspect in which the breakthroughs are mostly governed by the simulation studies, ${ }^{41,42}$ and the experimental findings and verifications usually lag due to difficulties associated with controlling the rate of nucleation and interface movements at nanoscale. Although, nanobubble interaction and interfacial phenomenon like Ostwald ripening, coalescence, ${ }^{43}$ fast transport of gases ${ }^{31}$ has been previously reported, the knowledge pertaining to the nanobubble interfacial mechanistic is limited. Thus, the importance of understanding the nanobubble interaction and the associated nanoscale phenomenon is manifold as it not only increases our knowledge about the fundamentals of nanobubble interactions, but also encourages the design of liquid-submerged novel nanoengineered surfaces, for example in two phase heat dissipation or in deep submergence mobility vehicle surfaces. Hence, imaging liquid interface between merging nanobubbles can provide further insights and understanding in gas transport phenomenon, thin films, and interfacial phenomenon at nanoscale.

In this work, focused study on the merging of two selected surface nanobubbles is carried out using LPEM. The region between the nanobubbles was evaluated based on the local image intensity on the TEM image, as the local intensity and image contrast reveals information on the mass and thickness of the specimen. ${ }^{44}$ During the observation, the interfacial distance between the nanobubbles was also tracked before initiation of merging. Further, the mechanism of merging is then proposed and evaluated in our study. We believe that understanding the merging phenomenon will aid to control and engineer the surface properties of surfaces submerged in liquids. 


\section{EXPERIMENTAL EQUIPMENT AND METHODS}

\section{Experimental Equipment}

The experiment was carried in a commercially available silicon nitride liquid cell electron microscope system (Poseidon Select, Protochips Inc., U.S.A.), as shown in Figure 1(i). It is an insitu electron microscopy platform which allows direct observation of liquids in TEM. In our study, $150 \mathrm{~nm}$ thick water film was sandwiched between two silicon based environmental chips (e-chips used: large e-chip (EHT-45ZZ, Protochips Inc., U.S.A.); small e-chip (EPB-55DS, Protochips Inc., U.S.A. )). These e-chips have a $50 \mathrm{~nm}$ thick silicon nitride $\left(\mathrm{SiN}_{\mathrm{x}}\right)$ windows suspended through a trapezoidal prism cavity of base dimensions $550 \mu \mathrm{m} \times 50 \mu \mathrm{m}$ for the in-situ observation. The echips were hydrophilized using a glow discharge plasma reactor (PIB-10, Vacuum Devices, Japan) before their assembly in the liquid cell holder. Moreover, the e-chips were assembled in crossaligned configuration so that their windows were perpendicular to each other and the available observation area was $50 \mu \mathrm{m} \times 50 \mu \mathrm{m}$. This was done to minimize the window bowing during the TEM observation. After assembling the liquid cell, it was kept in a vacuum pumping station $\left(10^{-}\right.$ ${ }^{3} \sim 10^{-4} \mathrm{~Pa}$ ) and water was allowed to flow between the e-chips for $\sim 12$ hours to ensure its integrity. After loading the sample into the high vacuum column of the TEM, the flow water was allowed to complete one cycle in order to eliminate any air cavities in the liquid cell. All the observations were carried in static condition with no flow inside the liquid cell. 

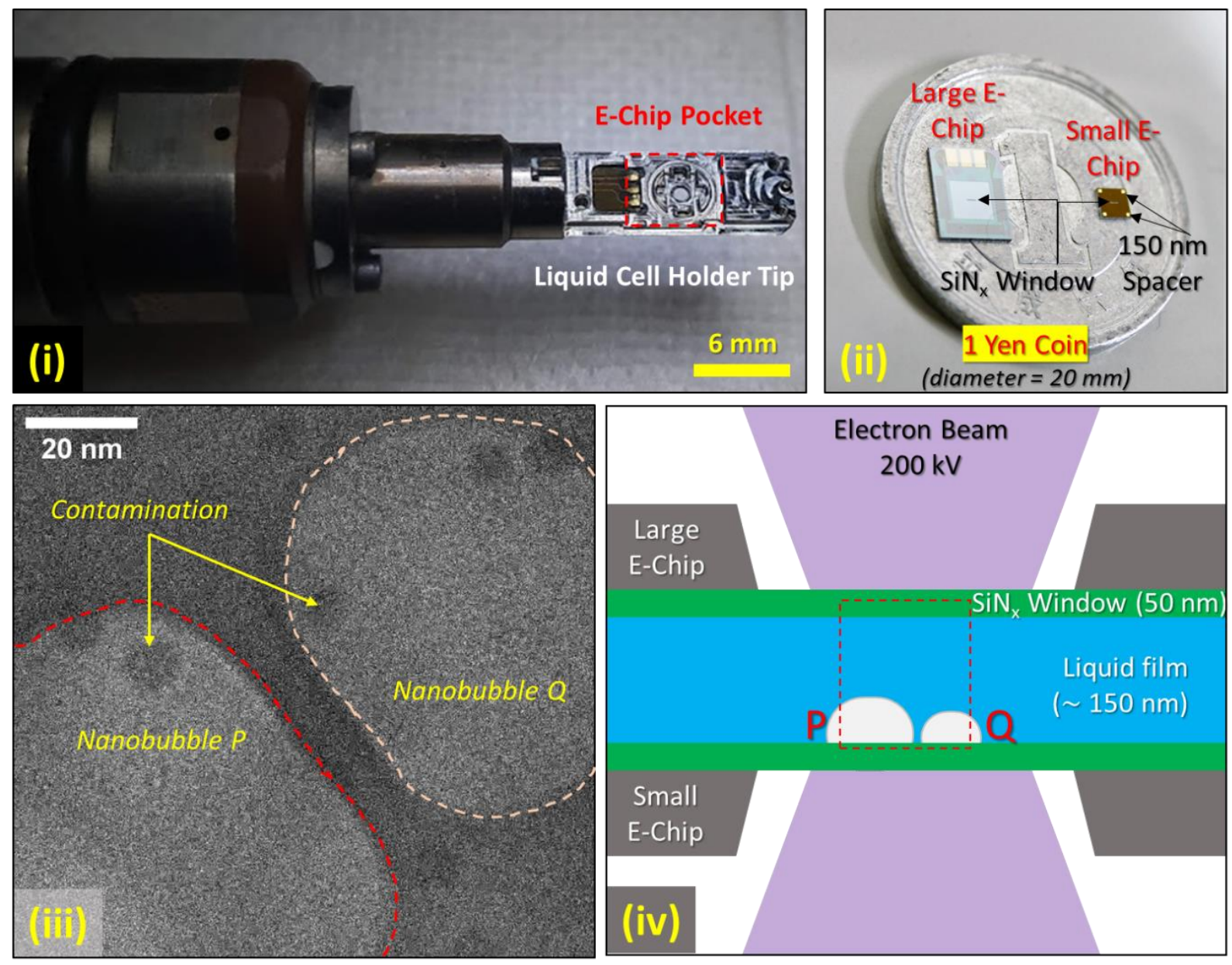

Figure 1. (i) Tip of the electron microscopy liquid cell holder. The red dashed line shows the pocket where E-chip are assembled for the liquid phase electron microscopy observation. (ii) Large E-chip and small E-chip placed on the 1 JPY coin (coin diameter: $20 \mathrm{~mm}$ ). The small E-chips has Su-8 spacers of height $150 \mathrm{~nm}$ at its corners to accommodate the liquid thin film. (iii) Image frame from the TEM experiment showing nanobubbles $\mathrm{P}$ and $\mathrm{Q}$ with initial radii $36 \mathrm{~nm}$ and $31 \mathrm{~nm}$, respectively, and having separation of $\sim 10-12 \mathrm{~nm}$ between their adjacent interfaces. Dark spots of size $\sim 5 \mathrm{~nm}$ represents the contamination possibly present on the adjacent $\mathrm{SiN}_{\mathrm{x}}$ window. The image is acquired at 250,000 magnification. The video of these nanobubbles before merging is available in Video S1. (iv) Schematic diagram of liquid cell and the surface nanobubbles during the 
observation. As highlighted using the red-dashed line, the region of interest is the area between the adjacent interfaces of two nanobubbles.

\section{TEM Imaging}

The sample was imaged in a TEM (JEM-2100Plus Electron Microscope, JEOL Ltd., Japan), operated at an accelerating voltage of $200 \mathrm{kV}$. This TEM has a thermionic electron emission source ( $\mathrm{LaB}_{6}$ filament), whose current density is three order less than the field emission electron sources, and hence has minimal beam effects on the sample. ${ }^{31}$ In addition to this, the study was carried using a higher spot size (Spot Size: 2-3). The spot size is controlled by the condenser lens of the TEM and increasing the spot size reduces the beam convergence as well as the beam current density irradiated on the sample. This is advantageous when samples are susceptible to high electron beam effects and avoids localized beam damage on the liquid cell. Hence, higher spot size sustains observation for longer time duration. The typical beam current density measured at the CCD camera sensor was $\sim 0.3 \mathrm{~A} / \mathrm{cm}^{2}$. The image acquisition was done using a CCD camera (Rio 16, Gatan Inc., U.S.A.) at $1 \mathrm{~K}$ resolution and 40 fps.

\section{Image Analysis}

Since the TEM image consists of varying grey levels depending on local mass or local thickness in the sample, using a systematic image processing methodology becomes highly relevant. Since the acquired phenomenon was quasistatic, the video initially acquired at $40 \mathrm{fps}$ was rendered at 8 fps (play speed $1 \mathrm{x}$ ) for trouble-free data handling. MATLAB was used for image handling and image pre-processing. After converting the RGB image to greyscale image, histogram equalization, gaussian smoothening filter and canny edge detection algorithm were deployed for 
contrast adjustment, background noise smoothening and detecting edges, respectively. The resultant binary image with boundaries was then fussed on the original RGB image and the distance measurements were done manually using an open source image processing and scientific image analysis package: Fiji ${ }^{45}$ Sequential snapshots of the image processing steps are provided in Supplementary Information (Figure S1). Qualitative estimation of the height profiles of the TEM images and their 3-D reconstruction based on image's local intensity was done using Gwyddion. ${ }^{46}$ It is also an open source multi-platform software which can be used for general height field and greyscale image processing.

\section{RESULTS AND DISCUSSION}

\section{Nanobubble observation in the liquid cell}

The interaction between the water and ionizing electron beam leads to the formation of various molecular and radical products, with $\mathrm{H}_{2}$ and $\mathrm{O}_{2}$ being the gaseous species (radiolysis). ${ }^{47}$ This electron beam governed radiolysis of water produces rapid statistical fluctuations in the local concentration of the product species imparting the required free energy for nucleation. ${ }^{48}$ Since the saturation concentration in of $\mathrm{H}_{2}(1.57 \mathrm{mg} / \mathrm{l})$ in water is far less than that of $\mathrm{O}_{2}(41.60 \mathrm{mg} / \mathrm{l}), \mathrm{H}_{2}$ molecules agglomerate quickly to form nanobubbles. Additionally, the heating effect due to interaction between the ionizing electron beam and water is typically in the range of $2-3^{\circ} \mathrm{C},{ }^{30}$ hence, the nucleated bubbles are not the vapor bubbles. 


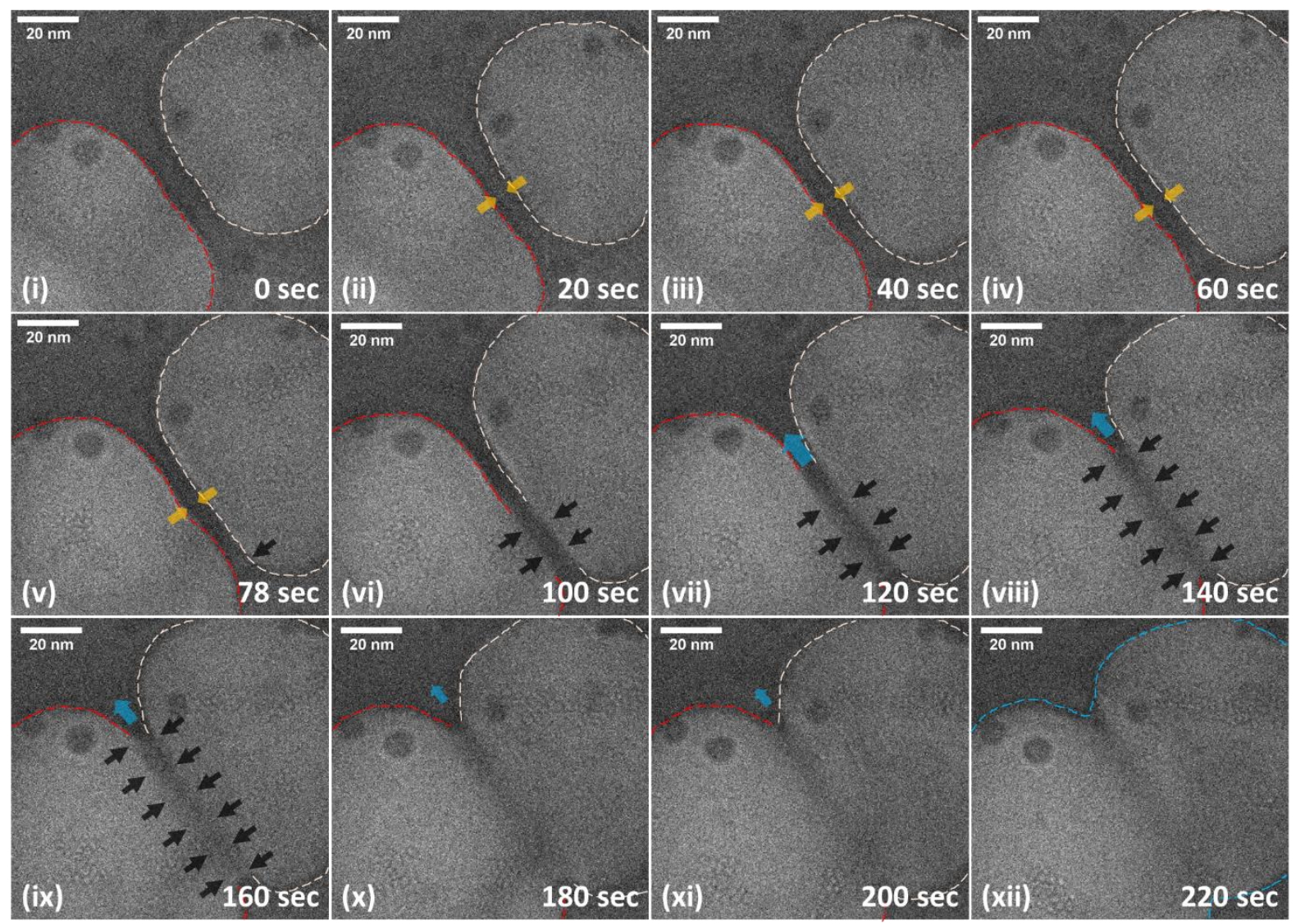

Figure 2. Time sequence of merging of nanobubbles observed using TEM for 220 seconds (i-xii). The interface of the nanobubbles is highlighted using the dashed-curved line. Yellow arrows show the bubble interface approaching during the initial phase. Black arrows show the increase in local image intensity between the nanobubbles. Blue arrow shows the increasing image intensity propagating in the plane parallel to the $\mathrm{SiN}_{\mathrm{x}}$ window. The video of nanobubbles before and during merging is available in Video $\mathrm{S} 1$ and $\mathrm{S} 2$, respectively.

Two stable surface nanobubbles (P and Q), were observed during the experiment (Figure 1(iii)). Since the contact area of nanobubbles $\mathrm{P}$ and $\mathrm{Q}$ was not circular, their radii, approximated using $\mathrm{r} \approx \sqrt{\mathrm{A} / \pi}$, and came out to be $36 \mathrm{~nm}$ and $31 \mathrm{~nm}$, respectively. These bubbles exhibited dark Fresnel fringe at their respective interfaces, therefore, it can be estimated that these bubbles are present on the lower $\mathrm{SiN}_{\mathrm{x}}$ window (region of under-focus). ${ }^{43}$ Moreover, the bubbles' contact angle, 
from the gas side, was estimated to be less than $90^{\circ}$ as no variation in the electron beam intensity was observed in the region outside the 3-phase contact line. For reference, a representative TEM image of nanobubbles exhibiting contact angle more than $90^{\circ}$ has been given in the supplementary information (Figure S2). Figure 1(iv) shows the schematic of the liquid cell as well as the TEM image of the nanobubbles. The observed nanobubbles were extremely stable in their form and showed a low initial growth rate ( $3 \mathrm{~nm}$ increase in radius in $\sim 80$ seconds). Although, after the initial nucleation, the experiment was carried with higher electron beam spot size to minimize the beam effects, the nanobubbles were observed to grow due to higher beam energy at relatively higher magnification (250,000X).

The continuous growth of the nanobubbles and the decreased interfacial distance created an opportunity to observe the merging of two surface nanobubbles with high spatial resolution. Figure 2 shows time resolved image sequence for the merging of these surface nanobubbles for 220 seconds of observation. The growth of the nanobubbles were observed for the initial 80 seconds of irradiation time (Figure 2(i-v)), followed by the initiation of the merging process of the interfaces (Figure 2(v-vi)). The variation in the separation between the adjacent interfaces of two nanobubbles was measured along the four segments (A, B, C, D) using the tuned Canny edge detection algorithm (Figure 3(i)). At the start of the observation, the separation between the two bubbles was in the range of $10-12 \mathrm{~nm}$. Due to the radial expansion of the interfaces, the separation gradually decreased up to the value of $\sim 6 \mathrm{~nm}$ (Figure 3(ii)). The segments $\mathrm{C}$ and D, where the initial separation was the lowest, were the fastest to obtain this separation value ( 100 seconds), followed by segments B and A (in that order). It is noteworthy that fluctuations were observed for all segments while approaching the minimum separation, as shown in the highlighted sections in Figure 3(ii). 


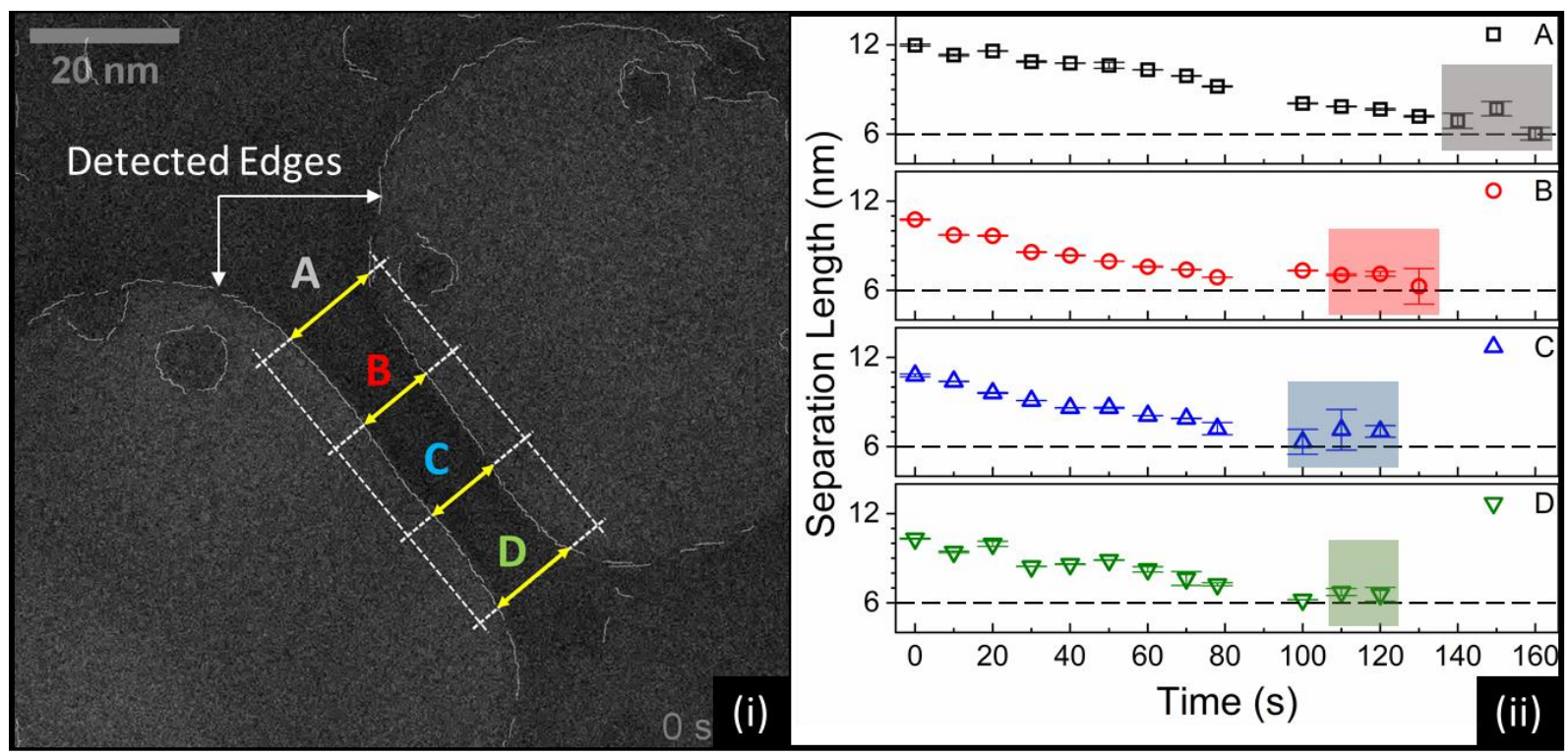

Figure 3. (i) Image, formed by overlapping the original RGB image with the edge detected binary image, showing the four specific segments, A, B, C and D, where the distance was measured (ii) Variation in the liquid film thickness between the nanobubbles $\mathrm{P}$ and $\mathrm{Q}$ measured at four distinguish segments. The highlighted region in each graph shows the fluctuations in the interfacial distance observed before merging.

At 100 seconds into the observation (Figure 2(vi)), the local image intensity of region between the two bubbles started to increase (as shown by black arrows). The variation in the local intensity suggests alteration in mass/thickness of the species in that particular region. ${ }^{44}$ This transformation then propagates to the remaining region between the bubble interfaces. In principle, human eye cannot detect the difference in images when the contrast variation is less than $10 \%$, therefore, the aid of image processing tool is essential to visualize and understand the actual phenomenon. ${ }^{44}$

To understand this image intensity variation and the evolution of liquid film between the two bubbles, a line plot based on the image intensity was plotted. Figure 4(i) shows the profile of the interfaces along the plane highlighted in the inset. The profiles were plotted for the merging initiation and completion phases, for the time 100-240 seconds. The profiles for the initial phase 
of nanobubble interaction has been provided in the supplementary information (Figure S3). It is important to note that the exact height of the bubbles could not be estimated, therefore, the Y-axis qualitatively represents the intensity of the image along the highlighted plane. A monotonic increase in image intensity was observed with time (Figure 4(ii)), suggesting the formation (initiation) and propagation (completion) of the species with lower mass/ thickness between two interfaces. We also confirmed similar growth in the region between the nanobubbles at four other planes corresponding to A, B, C and D (as in Figure 3(i)), provided in supplementary information (Figure S4).
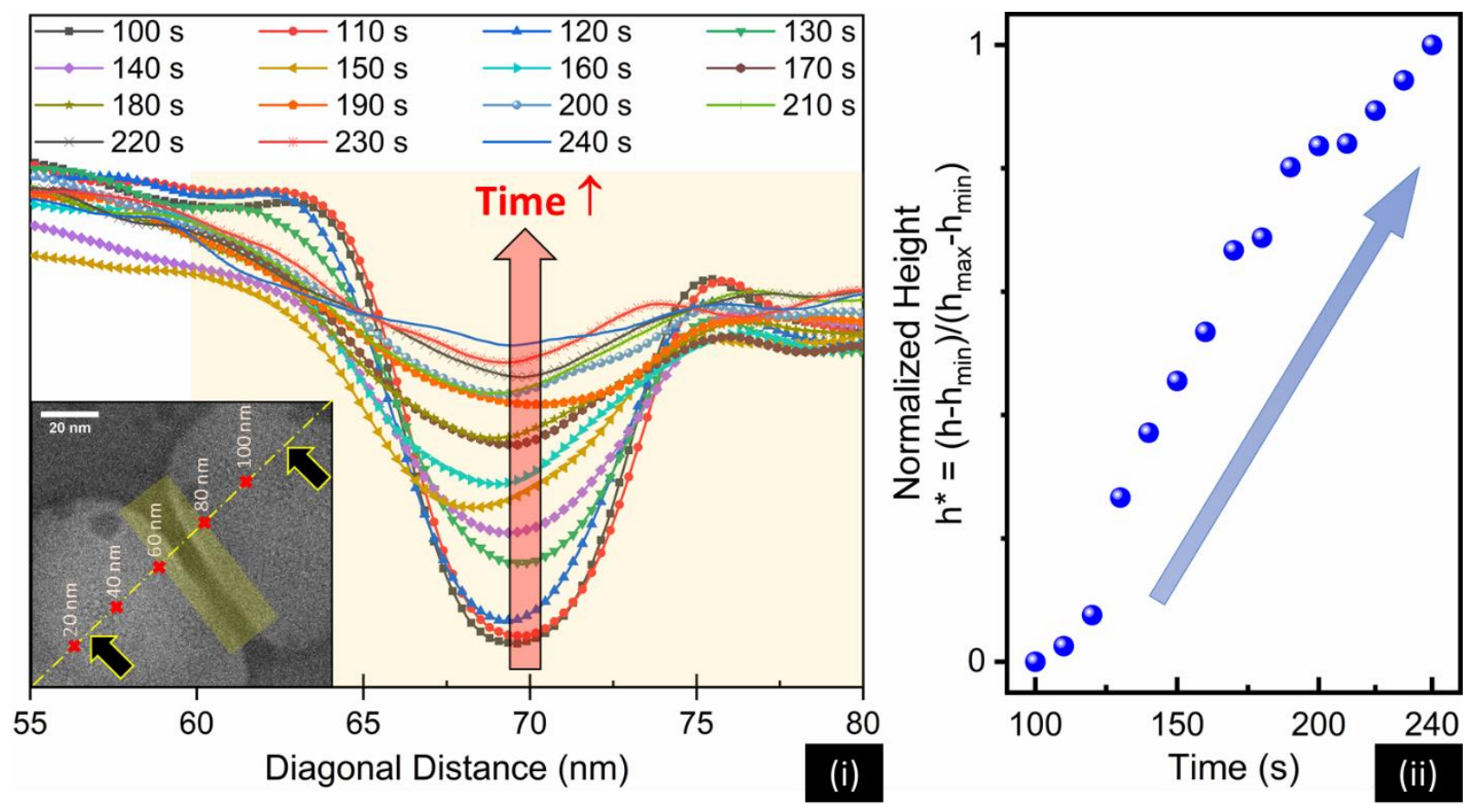

Figure 4. (i) Estimated profile of the region between the merging nanobubbles based on the variation in image intensity. The evolution of the intensity is shown for the time $100 \mathrm{~s}$ to $240 \mathrm{~s}$. Inset shows the plane along which the profile was plotted. (ii) Measured normalized height of the gas layer shows the monotonic increasing trend with time. 


\section{Insights on varying intensity between the bubbles}

The absence of supplemental invasive investigatory techniques to physically capture thin film with high resolution time scales has limited our capacity to filter down our findings, however, the change in the local intensity of the TEM image usually develops due the change in local density or the thickness of the region in study. One perspective of analyzing the change in local intensity is the variation of density of liquid confined between the interface. The presence of two bubbles in close vicinity may further strengthen this argument due to possibility of enhanced exchanges among them. However, various studies suggest that the inhomogeneity across the liquid surface is usually few Angstroms (molecular level), ${ }^{49,50}$ which is one order lower than the separation between two bubbles. The feasibility to capture such variation in liquid density in localized region with the TEM would be impossible. Additionally, hydration layers are responsible for density variations near the substrate surface, ${ }^{51,52}$ but the thickness of hydration layers (order of $10^{0} \mathrm{~nm}$ ) is too less to be practically captured in a $150 \mathrm{~nm}$ thick liquid using TEM.

The interaction between the two surface nanobubbles is often weak due to the presence of the three-phase contact line. ${ }^{53}$ However, for the slowly growing nanobubble pair (due to continuous e-beam exposure), the nanobubbles are destined to interact as their separation reduces. To understand their interaction, 3D reconstructions of the nanobubbles were done based on the intensity of the liquid and gas phases in the image. Figure 5 shows the TEM images as well as their reconstructed 3D images for time $t=0 \mathrm{~s}, 50 \mathrm{~s}, 100 \mathrm{~s}, 150 \mathrm{~s}, 200 \mathrm{~s}$ and $245 \mathrm{~s}$. It is important to note that the contaminations, highlighted in Figure 1(iii), present on the other $\operatorname{SiN}_{\mathrm{x}}$ window, were translated into hollow structures due the image processing algorithms for 3D reconstruction (due to their dark contrast). These 3D artifacts have been highlighted in Figure 5(i). On analyzing the contrast of the region between the two nanobubbles, one aspect of looking into the increase in the 
local intensity is the formation and increase in thickness of the gas film. At $\mathrm{t}=100 \mathrm{~s}$ (Figure 5(iii)), this change in intensity is quite noticeable. It can be construed from the reconstructed 3D image (based on changing thickness contrast) that a thin gas layer has formed. The formation of this gas layer initiates at the region having minimum separation $(\sim 6 \mathrm{~nm})$. At $t=100 \mathrm{~s}$, the formation of gas layer begins, as depicted by the purple arrows in Figure 5(iii), however, no formation is observed at the region of separation greater than $6 \mathrm{~nm}$ (blue arrows in Figure 5(iii)). The formation of the gas film acts as a precursor for the formation of bridge between two bubbles. The gas layer then advances in both radial as well as in perpendicular direction, as shown by red and purple arrows, respectively, in Figure 5(iv). The formation propagates and starts growth towards bulk liquid (Figure 5(v-vi)) which completes the merging process.

In addition, we also observed that the initial bubbles itself and then the merged bubble were not in perfectly hemispherical shape. Similar structures, flat gaseous enriched domains called micro/nano-pancakes, has been previously studied, both experimentally and using MD simulations. ${ }^{38,54-56}$ A recent MD simulation study on the two equal sized pinned nanobubbles suggested that the strong gas-solid interactions promotes the gas exchanges through tunneling along the solid surface ${ }^{53}$ Hence, the formation of gas layer may resemble gaseous enrichments on the surface which promotes merging of the nanobubble. Additionally, presence of ultra-high dense gas phase,${ }^{57}$ specifically, near the solid-gas interface inside a nanobubble ${ }^{58}$ might also promote the formation of gas layer between two nanobubbles. 


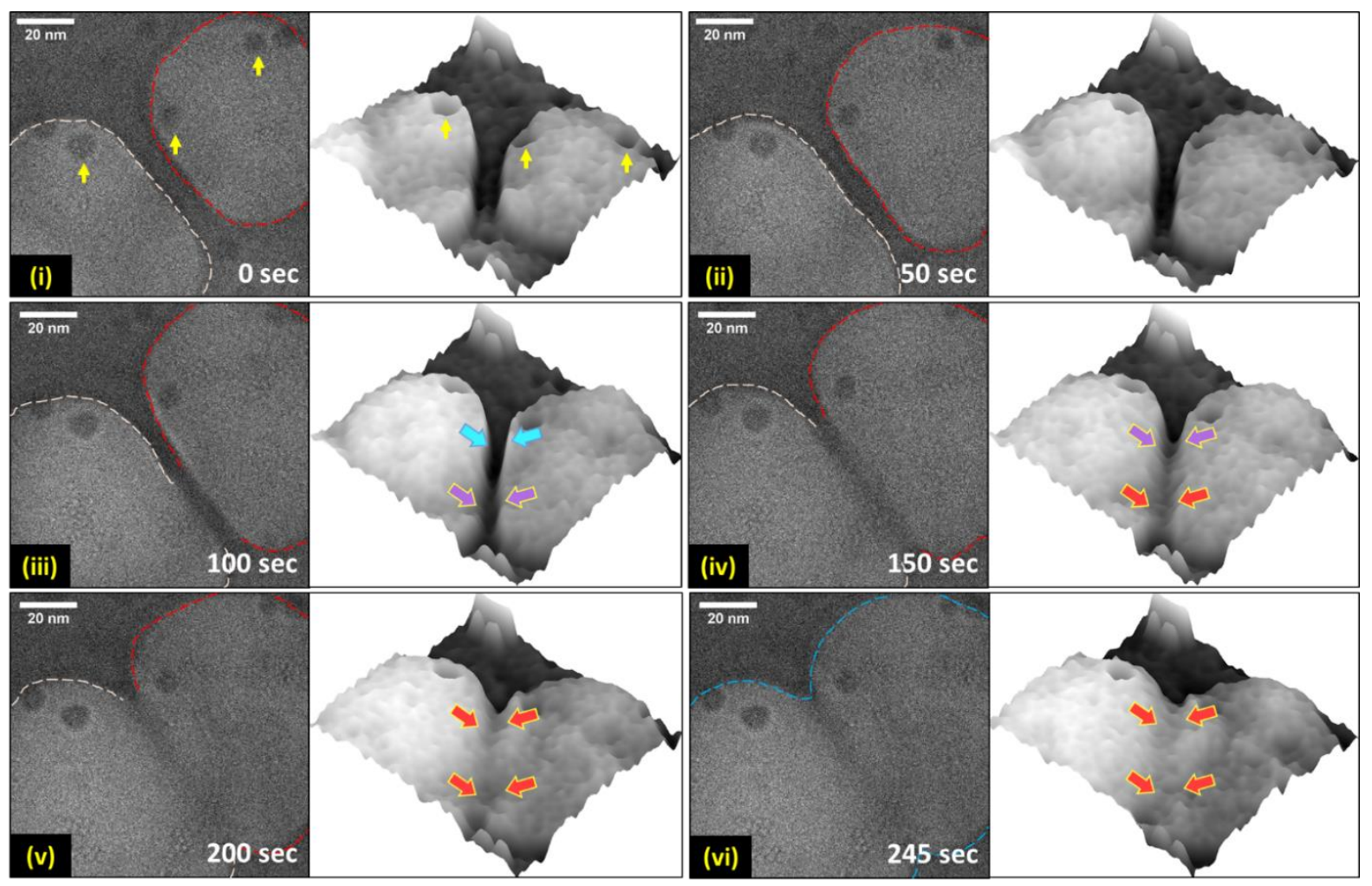

Figure 5. 3D reconstruction of 2D TEM images on basis of thickness contrast. Images are shown for (i) $0 \mathrm{~s}$; (ii) $50 \mathrm{~s}$; (iii) $100 \mathrm{~s}$; (iv) $150 \mathrm{~s}$; (v) $200 \mathrm{~s}$; and (vi) $245 \mathrm{~s}$. The yellow arrows in (i) highlights the contaminations which have been translated into hollow structures (artifacts) due the image processing algorithms for 3D reconstruction on nanobubbles. The purple arrows (in iii and iv) show the initiation of the formation of the gas layer at regions where the separation was minimum, whereas the blue arrows (in iii) shows the regions where the formation has not started yet. The red arrows (in iv, $\mathbf{v}$ and vi), show the growing gas layer in the direction perpendicular to the gas-solid interface.

Moreover, it is evident from the process time scales that the interaction is very weak when the effects of electron beam are reduced to a minimal. To estimate the effect of electron beam and the saturation levels on the formation of gas film, we carried a similar experiment but at stronger electron beam conditions $\left(\sim 2.8 \mathrm{~A} / \mathrm{cm}^{2}\right)$ and observed the phenomenon to be at least one order of 
magnitude faster. This indicates that the rate of the gas film formation and growth is strongly dependent on the gas saturation. The observation at higher electron beam density and their 3D reconstructions are given in supplementary information (Figure S5). The merged nanobubble also has a neck formed due to the merging. Similar shaped nanobubbles have been observed previously using AFM, ${ }^{26}$ however AFM's lack of in-situ capabilities possibly prevented appreciation of such

a phenomenon. A similar proposition has been put forward using MD simulations ${ }^{41}$ for unpinned surface nanobubbles, where the authors have discussed on formation of a layer of gas molecules, on solid surface with high gas-solid interaction, which serves as a bridge for the exchange between the nanobubbles. Since the surface bubbles observed in our experiments were pinned, the perturbations necessary for the nanobubble interaction was provided by the electron beam.

\section{Mechanism for the formation of thin gas layer}

The formation of the gas layer mechanism can be explained by the following pathway (Figure 6). The gas molecules formed due to low but continuous electron irradiation leads to the initial quasistatic growth of nanobubbles (Figure 6(i)). Moreover, this irradiation also creates a higher local saturation of gas molecules through its path. As the nanobubbles' adjacent interfacial distance decreases, the number of the gas molecules in the region close to the adjacent three phase contact lines grows which increases the effective gas molecular density in this region. The increased local gas concentration leads to the accumulation of molecules and ultimately leads to the formation of a thin gas layer near the surface (Figure 6(ii)). This gas layer exhibits very slow growth dynamics because of the intrinsic strong liquid-solid interaction of the window material. The gas layer gradually expands and spreads throughout the region in-between nanobubbles, thus completing the merging of the nanobubbles (Figure 6(iii)). 

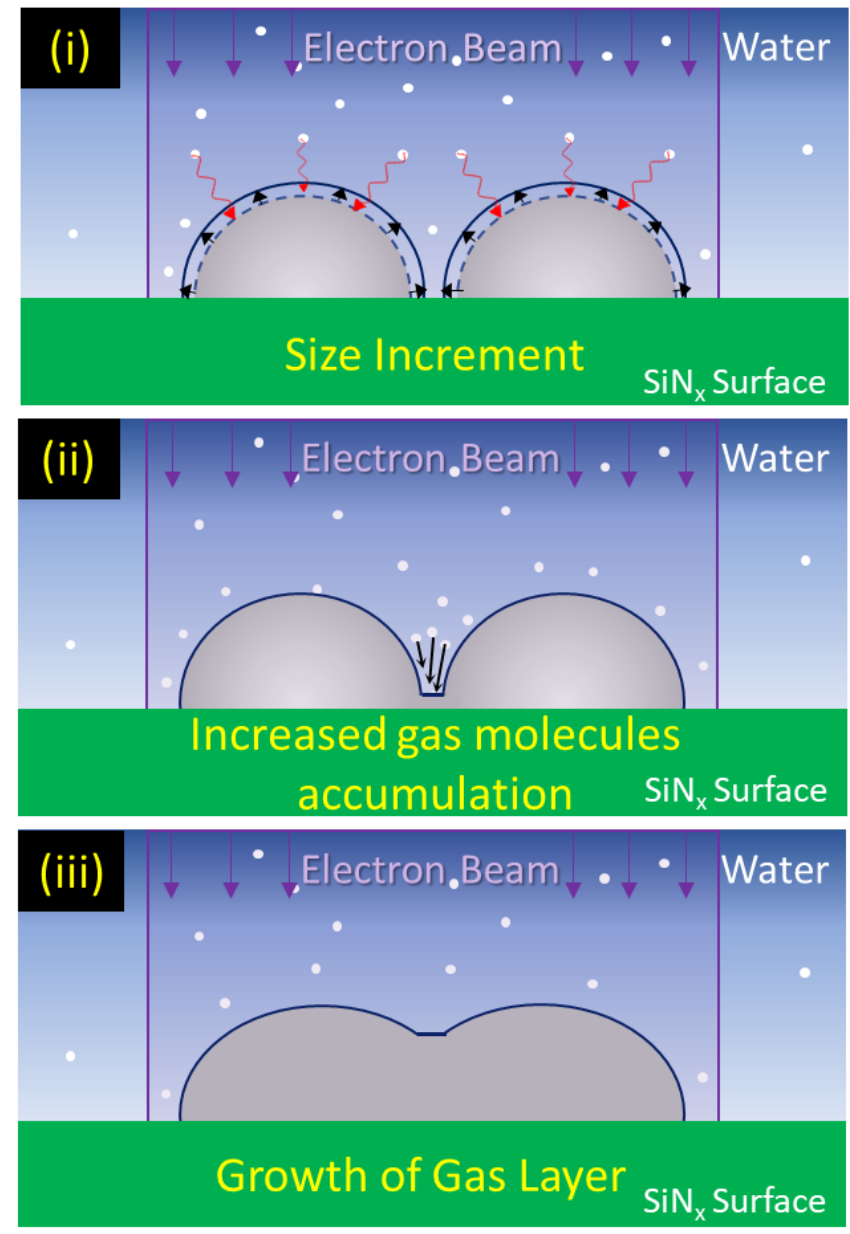

Figure 6. Pathway for the formation of gas layer between two stable surface nanobubbles. (i) The electron beam increases the gas concentration of the irradiated region, leading the bubbles to expand. (ii) The gas molecules accumulate near the solid surface between the two bubbles, which increases the local gas concentration. (iii) The nucleated gas layer grows while acting as a bridge between the two nanobubbles.

Our proposition is further supported by the fluctuations of interfaces observed when the separation approached to its minimum (Figure 3(ii)). We believe that the fluctuations are due to the shifting Fresnel fringes when thin gas layer is formed. The formation of the thin gas layer on the surface interferes with the interface of bubble imaging in a way that apparent increase in the separation is observed. 


\section{CONCLUSION}

In summary, we studied the merging of two stable surface nanobubbles using in-situ liquid phase electron microscopy. Analysis of the TEM's contrast images and their intensity profiles helped in understanding the dynamics of formation of gas layers and the successive nanobubble merging. We found that the formation of the thin film initiated in a localized region-wise manner when the interfacial distance between the adjacent nanobubbles decreased. We also propose a plausible mechanism indicating the formation of high gas molecule dense region between the nanobubbles. These highly saturated regions were postulated to initiate the formation of gas layer at the regions where the separation between the nanobubbles was minimum. Our results suggest the formation of the bridge between the stable surface nanobubbles which is important for their merging. Future studies on this subject can be focused on the slow evolution of gas layer, its precise measurements, and the quantitative evaluation of liquid surface interactions, as well as on the estimation of gas saturation and the successive coalescence of nanobubbles at different saturation levels. Nonetheless, the present analysis serves as prima facie affirmation of the bubble merging process.

\section{Associated Content}

\section{Supplementary Information}

The following files are available free of charge.

Image Processing methodology and their snapshots at various intermediate steps, reference TEM image of nanobubbles having contact angle above $90^{\circ}$, estimated profile traces of the region 
between the nanobubbles for the initial phase and at various segments of the image, nanobubble merging observed at high electron beam intensity.

Nanobubbles imaged before the initiation of merging process (Video S1).

Nanobubbles imaged during the merging (Video S2).

\section{Author Information}

\section{Corresponding Author}

Masamichi Kohno - Department of Mechanical Engineering; and International Institute for Carbon-Neutral Energy Research (WPI-I2CNER), Kyushu University, Fukuoka 819-0395, Japan *Email: kohno@mech.kyushu-u.ac.jp

\section{Authors}

Sarthak Nag - Department of Mechanical Engineering; and International Institute for CarbonNeutral Energy Research (WPI-I2CNER), Kyushu University, Fukuoka 819-0395, Japan

Yoko Tomo - Department of Mechanical Engineering; and International Institute for CarbonNeutral Energy Research (WPI-I2CNER), Kyushu University, Fukuoka 819-0395, Japan

Koji Takahashi - Department of Aeronautics and Astronautics; and International Institute for Carbon-Neutral Energy Research (WPI-I2CNER), Kyushu University, Fukuoka 819-0395, Japan

\section{Author Contributions}

The manuscript was written through contributions of all authors. All authors have given approval to the final version of the manuscript.

\section{Funding Sources}


This work was financially supported by the Core Research for Evolutional Science and Technology project of Japan Science and Technology Agency (JST-CREST) Grant Number JPMJCR18I1 and the Japanese Society for the Promotion of Science (JSPS) KAKENHI Grant Numbers JP20J13061 and JP19K23490.

\section{Acknowledgements}

The authors gratefully acknowledge Dr. Hideaki Teshima (Osaka University, Japan) for his suggestions and technical discussions. The authors also acknowledge the financially support of the Core Research for Evolutional Science and Technology project of Japan Science and Technology Agency (JST-CREST) Grant Number JPMJCR18I1 and the Japanese Society for the Promotion of Science (JSPS) KAKENHI Grant Numbers JP20J13061 and JP19K23490.

\section{References}

(1) Xie, L.; Cui, X.; Gong, L.; Chen, J.; Zeng, H. Recent Advances in the Quantification and Modulation of Hydrophobic Interactions for Interfacial Applications. Langmuir 2020, 36 (12), 2985-3003. https://doi.org/10.1021/acs.langmuir.9b03573.

(2) Nazari, M.; Nazifi, S.; Huang, Z.; Tong, T.; Ouro-Koura, H.; Bao, J.; Das, K.; Ghasemi, H. Surface Tension Nanogates for Controlled Ion Transport. ACS Appl. Nano Mater. 2020, 3 (7), 6979-6986. https://doi.org/10.1021/acsanm.0c01304.

(3) Yan, M.; Li, T.; Zheng, P.; Wei, R.; Jiang, Y.; Li, H. Wetting State Transition of a Liquid Gallium Drop at the Nanoscale. Phys. Chem. Chem. Phys. 2020, 22 (21), 11809-11816. https://doi.org/10.1039/d0cp00985g. 
(4) Lohse, D.; Zhang, X. Surface Nanobubbles and Nanodroplets. Rev. Mod. Phys. 2015, 87 (3), 981. https://doi.org/10.1103/RevModPhys.87.981.

(5) Ghaani, M. R.; Kusalik, P. G.; English, N. J. Massive Generation of Metastable Bulk Nanobubbles in Water by External Electric Fields. Sci. Adv. 2020, 6 (14), eaaz0094. https://doi.org/10.1126/sciadv.aaz0094.

(6) Seddon, J. R. T.; Lohse, D. Nanobubbles and Micropancakes: Gaseous Domains on Immersed Substrates. J. Phys. Condens. Matter 2011, 23 (13), 133001. https://doi.org/10.1088/0953-8984/23/13/133001.

(7) Zhang, X.; Lohse, D. Perspectives on Surface Nanobubbles. Biomicrofluidics 2014, 8 (4), 134702. https://doi.org/10.1063/1.4891097.

(8) Kurita, Y.; Chiba, I.; Kijima, A. Physical Eradication of Small Planktonic Crustaceans from Aquaculture Tanks with Cavitation Treatment. Aquac. Int. 2017, 25 (6), 2127-2133. https://doi.org/10.1007/s10499-017-0179-1.

(9) Agarwal, A.; Ng, W. J.; Liu, Y. Principle and Applications of Microbubble and Nanobubble Technology for Water Treatment. Chemosphere 2011, 84 (9), 1175-1180. https://doi.org/10.1016/j.chemosphere.2011.05.054.

(10) Atkinson, A. J.; Apul, O. G.; Schneider, O.; Garcia-Segura, S.; Westerhoff, P. Nanobubble Technologies Offer Opportunities To Improve Water Treatment. Acc. Chem. Res. 2019, 52 (5), 1196-1205. https://doi.org/10.1021/acs.accounts.8b00606.

(11) Yang, S.; Duisterwinkel, A. Removal of Nanoparticles from Plain and Patterned Surfaces Using Nanobubbles. Langmuir 2011, $27 \quad$ (18), 11430-11435. 
https://doi.org/10.1021/la2010776.

(12) Li, C.; Zhang, A. M.; Wang, S.; Cui, P. Formation and Coalescence of Nanobubbles under Controlled Gas Concentration and Species. AIP Adv. 2018, 8 (1), 015104. https://doi.org/10.1063/1.5013244.

(13) Batchelor, D. V. B.; Abou-Saleh, R. H.; Coletta, P. L.; McLaughlan, J. R.; Peyman, S. A.; Evans, S. D. Nested Nanobubbles for Ultrasound-Triggered Drug Release. ACS Appl. Mater. Interfaces 2020, 12 (26), 29085-29093. https://doi.org/10.1021/acsami.0c07022.

(14) Wu, Z. H.; Chen, H. B.; Dong, Y. M.; Mao, H. L.; Sun, J. L.; Chen, S. F.; Craig, V. S. J.; Hu, J. Cleaning Using Nanobubbles: Defouling by Electrochemical Generation of Bubbles. J. Colloid Interface Sci. 2008, 328 (1), 10-14. https://doi.org/10.1016/j.jcis.2008.08.064.

(15) Kang, E.; Min, H. S.; Lee, J.; Han, M. H.; Ahn, H. J.; Yoon, I.-C.; Choi, K.; Kim, K.; Park, K.; Kwon, I. C. Nanobubbles from Gas-Generating Polymeric Nanoparticles: Ultrasound Imaging of Living Subjects. Angew. Chemie Int. Ed. 2010, 49 (3), 524-528. https://doi.org/10.1002/anie.200903841.

(16) Li, J.; Xi, A.; Qiao, H.; Liu, Z. Ultrasound-Mediated Diagnostic Imaging and Advanced Treatment with Multifunctional Micro/Nanobubbles. Cancer Lett. 2020, 475, 92-98. https://doi.org/10.1016/j.canlet.2020.01.028.

(17) Fan, Y. W.; Wang, R. Z. Submicrometer-Sized Vaterite Tubes Formed Through Nanobubble-Templated Crystal Growth. Adv. Mater. 2005, 17 (19), 2384-2388. https://doi.org/10.1002/adma.200500755.

(18) Zhou, Y.; Bastida, F.; Zhou, B.; Sun, Y.; Gu, T.; Li, S.; Li, Y. Soil Fertility and Crop 
Production Are Fostered by Micro-Nano Bubble Irrigation with Associated Changes in Soil Bacterial Community. Soil Biol. Biochem. 2020, 141, 107663. https://doi.org/10.1016/j.soilbio.2019.107663.

(19) Zou, J.; Zhang, H.; Guo, Z.; Liu, Y.; Wei, J.; Huang, Y.; Zhang, X. Surface Nanobubbles Nucleate Liquid Boiling. Langmuir 2018, 34 (46), 14096-14101. https://doi.org/10.1021/acs.langmuir.8b03290.

(20) Zhang, X.; Lhuissier, H.; Sun, C.; Lohse, D. Surface Nanobubbles Nucleate Microdroplets. Phys. Rev. Lett. 2014, 112 (14), 144503. https://doi.org/10.1103/PhysRevLett.112.144503.

(21) Wang, Y.; Bhushan, B.; Zhao, X. Improved Nanobubble Immobility Induced by Surface Structures on Hydrophobic Surfaces. Langmuir 2009, 25 (16), 9328-9336. https://doi.org/10.1021/la901186a.

(22) Attard, P. The Stability of Nanobubbles. Eur. Phys. J. Spec. Top. 2013, 1-22. https://doi.org/10.1140/epjst/e2013-01817-0.

(23) Sun, Y.; Xie, G.; Peng, Y.; Xia, W.; Sha, J. Stability Theories of Nanobubbles at SolidLiquid Interface: A Review. Colloids Surfaces A Physicochem. Eng. Asp. 2016, 495, 176186. https://doi.org/10.1016/j.colsurfa.2016.01.050.

(24) Ducker, W. A. Contact Angle and Stability of Interfacial Nanobubbles. Langmuir 2009, 25 (16), 8907-8910. https://doi.org/10.1021/1a902011v.

(25) Ushikubo, F. Y.; Furukawa, T.; Nakagawa, R.; Enari, M.; Makino, Y.; Kawagoe, Y.; Shiina, T.; Oshita, S. Evidence of the Existence and the Stability of Nano-Bubbles in Water. Colloids Surfaces A Physicochem. Eng. Asp. 2010, 361 (1-3), 31-37. 
https://doi.org/10.1016/j.colsurfa.2010.03.005.

(26) Teshima, H.; Nishiyama, T.; Takahashi, K. Nanoscale Pinning Effect Evaluated from Deformed Nanobubbles. J. Chem. Phys. 2017, 146 (1), 014708. https://doi.org/10.1063/1.4973385.

(27) Weijs, J. H.; Lohse, D. Why Surface Nanobubbles Live for Hours. Phys. Rev. Lett. 2013, 110 (5), 054501. https://doi.org/10.1103/PhysRevLett.110.054501.

(28) Brenner, M. P.; Lohse, D. Dynamic Equilibrium Mechanism for Surface Nanobubble $\begin{array}{lllllll}\text { Stabilization. } & \text { Phys. Rev. Lett. 2008, } 101 & \text { (21), } 214505 .\end{array}$ https://doi.org/10.1103/PhysRevLett.101.214505.

(29) Zhang, J.; Lin, L.; Sun, L.; Huang, Y.; Koh, A. L.; Dang, W.; Yin, J.; Wang, M.; Tan, C.; Li, T.; Tan, Z.; Liu, Z.; Peng, H. Clean Transfer of Large Graphene Single Crystals for High-Intactness Suspended Membranes and Liquid Cells. Adv. Mater. 2017, 29 (26), 1700639. https://doi.org/10.1002/adma.201700639.

(30) Jensen, E.; Mølhave, K. Encapsulated Liquid Cells for Transmission Electron Microscopy. In Liquid Cell Electron Microscopy; Ross, F. M., Ed.; Cambridge University Press, 2016; pp 35-55.

(31) Shin, D.; Park, J. B.; Kim, Y. J.; Kim, S. J.; Kang, J. H.; Lee, B.; Cho, S. P.; Hong, B. H.; Novoselov, K. S. Growth Dynamics and Gas Transport Mechanism of Nanobubbles in Graphene Liquid Cells. Nat. Commun. 2015, 6 (1), 1-6. https://doi.org/10.1038/ncomms7068.

(32) Lou, S.; Gao, J.; Xiao, X.; Li, X.; Li, G.; Zhang, Y.; Li, M.; Sun, J.; Li, X.; Hu, J. Studies 
of Nanobubbles Produced at Liquid/Solid Interfaces. In Materials Characterization; Elsevier, 2002; Vol. 48, pp 211-214. https://doi.org/10.1016/S1044-5803(02)00241-3.

(33) Chan, C. U.; Ohl, C. D. Total-Internal-Reflection-Fluorescence Microscopy for the Study of Nanobubble Dynamics. Phys. Rev. Lett. 2012, 109 (17), 174501. https://doi.org/10.1103/PhysRevLett.109.174501.

(34) Dyett, B. P.; Zhang, X. Accelerated Formation of H2Nanobubbles from a Surface Nanodroplet Reaction. ACS Nano 2020, $14 \quad$ (9), 10944-10953. https://doi.org/10.1021/acsnano.0c03059.

(35) Yang, J.; Alam, S. B.; Yu, L.; Chan, E.; Zheng, H. Dynamic Behavior of Nanoscale Liquids in Graphene Liquid Cells Revealed by in Situ Transmission Electron Microscopy. Micron 2019, 116, 22-29. https://doi.org/10.1016/j.micron.2018.09.009.

(36) Tomo, Y.; Li, Q. Y.; Ikuta, T.; Takata, Y.; Takahashi, K. Unexpected Homogeneous Bubble Nucleation near a Solid-Liquid Interface. J. Phys. Chem. C 2018, 122 (50), 28712-28716. https://doi.org/10.1021/acs.jpcc.8b09200.

(37) Kim, Qh.; Shin, D.; Park, J.; Weitz, D. A.; Jhe, W. Initial Growth Dynamics of $10 \mathrm{Nm}$ Nanobubbles in the Graphene Liquid Cell. Appl. Nanosci. 2018, 0, 3. https://doi.org/10.1007/s13204-018-0925-3.

(38) Huang, T. W.; Liu, S. Y.; Chuang, Y. J.; Hsieh, H. Y.; Tsai, C. Y.; Wu, W. J.; Tsai, C. T.; Mirsaidov, U.; Matsudaira, P.; Chang, C. S.; Tseng, F. G.; Chen, F. R. Dynamics of Hydrogen Nanobubbles in KLH Protein Solution Studied with in Situ Wet-TEM. Soft Matter 2013, 9 (37), 8856-8861. https://doi.org/10.1039/c3sm50906k. 
(39) White, E. R.; Mecklenburg, M.; Singer, S. B.; Aloni, S.; Regan, B. C. Imaging Nanobubbles in Water with Scanning Transmission Electron Microscopy. Appl. Phys. Express 2011, 4 (5), 055201. https://doi.org/10.1143/APEX.4.055201.

(40) Park, J. B.; Shin, D.; Kang, S.; Cho, S. P.; Hong, B. H. Distortion in Two-Dimensional Shapes of Merging Nanobubbles: Evidence for Anisotropic Gas Flow Mechanism. Langmuir 2016, 32 (43), 11303-11308. https://doi.org/10.1021/acs.langmuir.6b01672.

(41) Li, C.; Wang, S. P.; Zhang, A. M.; Liu, Y. Dynamic Behavior of Two Neighboring Nanobubbles Induced by Various Gas-Liquid-Solid Interactions. Phys. Rev. Fluids 2018, 3 (12), 123604. https://doi.org/10.1103/PhysRevFluids.3.123604.

(42) German, S. R.; Wu, X.; An, H.; Craig, V. S. J.; Mega, T. L.; Zhang, X. Interfacial Nanobubbles Are Leaky: Permeability of the Gas/Water Interface. ACS Nano 2014, 8 (6), 6193-6201. https://doi.org/10.1021/nn5016049.

(43) Tomo, Y.; Takahashi, K.; Nishiyama, T.; Ikuta, T.; Takata, Y. Nanobubble Nucleation Studied Using Fresnel Fringes in Liquid Cell Electron Microscopy. Int. J. Heat Mass Transf. 2017, 108, 1460-1465. https://doi.org/10.1016/j.ijheatmasstransfer.2017.01.013.

(44) Williams, D. B.; Carter, C. B. Transmission Electron Microscopy-A Textbook for Materials Science, 2nd ed.; Springer US, 2009; pp 371-387.

(45) Schindelin, J.; Arganda-Carreras, I.; Frise, E.; Kaynig, V.; Longair, M.; Pietzsch, T.; Preibisch, S.; Rueden, C.; Saalfeld, S.; Schmid, B.; Tinevez, J. Y.; White, D. J.; Hartenstein, V.; Eliceiri, K.; Tomancak, P.; Cardona, A. Fiji: An Open-Source Platform for BiologicalImage Analysis. Nat. Methods 2012, 9 (7), 676-682. https://doi.org/10.1038/nmeth.2019. 
(46) Nečas, D.; Klapetek, P. Gwyddion: An Open-Source Software for SPM Data Analysis. Cent. Eur. J. Phys. 2012, 10 (1), 181-188. https://doi.org/10.2478/s11534-011-0096-2.

(47) Schneider, N. M.; Norton, M. M.; Mendel, B. J.; Grogan, J. M.; Ross, F. M.; Bau, H. H. Electron-Water Interactions and Implications for Liquid Cell Electron Microscopy. J. Phys. Chem. C 2014, 118 (38), 22373-22382. https://doi.org/10.1021/jp507400n.

(48) Bowers, P. G.; Hofstetter, C.; Letter, C. R.; Toomey, R. T. Supersaturation Limit for Homogeneous Nucleation of Oxygen Bubbles in Water at Elevated Pressure: “Superhenry's Law.” J. Phys. Chem. 1995, 99 (23), 9632-9637. https://doi.org/10.1021/j100023a048.

(49) Hill, T. L. An Introduction to Statistical Thermodynamics; Dover Publications, 1986; pp $301-320$.

(50) Guermeur, R.; Biquard, F.; Jacolin, C. Density Profiles and Surface Tension of Spherical Interfaces. Numerical Results for Nitrogen Drops and Bubbles. J. Chem. Phys. 1984, 82 (4), 2040-2051. https://doi.org/10.1063/1.448389.

(51) Yang, C. W.; Miyazawa, K.; Fukuma, T.; Miyata, K.; Hwang, I. S. Direct Comparison between Subnanometer Hydration Structures on Hydrophilic and Hydrophobic Surfaces via Three-Dimensional Scanning Force Microscopy. Phys. Chem. Chem. Phys. 2018, 20 (36), 23522-23527. https://doi.org/10.1039/c8cp02309c.

(52) Teshima, H.; Li, Q. Y.; Takata, Y.; Takahashi, K. Gas Molecules Sandwiched in Hydration Layers at Graphite/Water Interfaces. Phys. Chem. Chem. Phys. 2020, 22 (24), 1362913636. https://doi.org/10.1039/d0cp01719a.

(53) Maheshwari, S.; Van Der Hoef, M.; Rodríguez Rodríguez, J.; Lohse, D. Leakiness of Pinned 
Neighboring Surface Nanobubbles Induced by Strong Gas-Surface Interaction. ACS Nano 2018, 12 (3), 2603-2609. https://doi.org/10.1021/acsnano.7b08614.

(54) Zhang, X. H.; Zhang, X.; Sun, J.; Zhang, Z.; Li, G.; Fang, H.; Xiao, X.; Zeng, X.; Hu, J. Detection of Novel Gaseous States at the Highly Oriented Pyrolytic Graphite-Water Interface. Langmuir 2007, 23 (4), 1778-1783. https://doi.org/10.1021/la062278w.

(55) Dammer, S. M.; Lohse, D. Gas Enrichment at Liquid-Wall Interfaces. Phys. Rev. Lett. 2006, 96 (20), 206101. https://doi.org/10.1103/PhysRevLett.96.206101.

(56) Teshima, H.; Takata, Y.; Takahashi, K. Adsorbed Gas Layers Limit the Mobility of $\begin{array}{lllllll}\text { Micropancakes. Appl. Phys. Lett. 2019, } 115 & \text { (7), } 071603 .\end{array}$ https://doi.org/10.1063/1.5113810.

(57) Zhou, L.; Wang, X.; Shin, H. J.; Wang, J.; Tai, R.; Zhang, X.; Fang, H.; Xiao, W.; Wang, L.; Wang, C.; Gao, X.; Hu, J.; Zhang, L. Ultrahigh Density of Gas Molecules Confined in Surface Nanobubbles in Ambient Water. J. Am. Chem. Soc. 2020, 142 (12), 5583-5593. https://doi.org/10.1021/jacs.9b11303.

(58) Wang, S.; Zhou, L.; Wang, X.; Wang, C.; Dong, Y.; Zhang, Y.; Gao, Y.; Zhang, L.; Hu, J. Force Spectroscopy Revealed a High-Gas-Density State near the Graphite Substrate inside Surface Nanobubbles. Langmuir 2019, $35 \quad$ (7), 2498-2505. https://doi.org/10.1021/acs.langmuir.8b03383. 
Table of Content/ Abstract Graphic $(8.5 \mathrm{~cm} \times 4.75 \mathrm{~cm})$

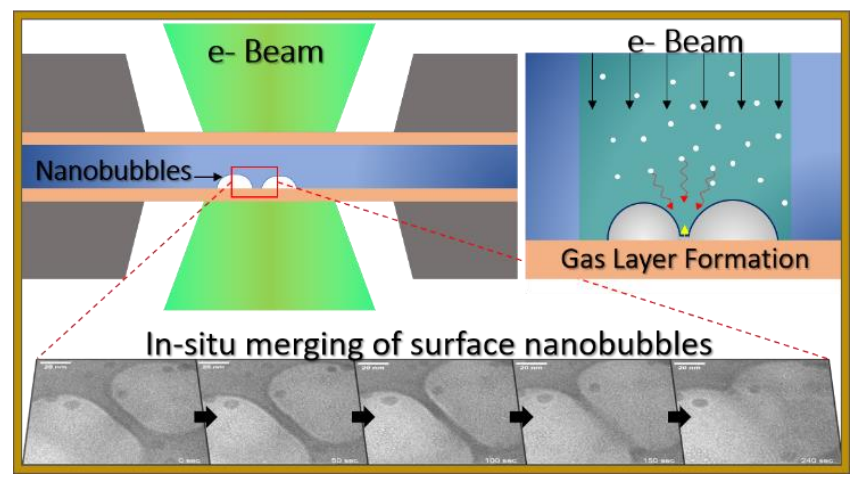

